\title{
Fibroma of Buccal Mucosa: Case Report and Literature Review
}

\section{Khaled Mohamed Afify Elrahawy (BDS, MD, PhD)}

Oral \& Maxillofacial Pathology Department, College of Dentistry, Al Jouf University, Saudi Arabia

Ahmed Ismail Nagy (BDS, MDS, ESOI)

Oral \& Maxillofacial Surgery Department, College of Dentistry, Al Jouf University, Saudi Arabia

Rayan Okla El-Swilem (BDS)

College of Dentistry, Al Jouf University, Saudi Arabia

doi: 10.19044/esj.2017.v13n6p267～ＵRL:http://dx.doi.org/10.19044/esj.2017.v13n6p267

\begin{abstract}
The fibroma, also referred to as irritation fibroma, is by far the most common of the oral fibrous tumorlike growths. While the terminology implies a benign neoplasm, most if not all fibromas represent reactive focal fibrous hyperplasia due to trauma or local irritation. Although the term focal fibrous hyperplasia more accurately describes the clinical appearance and pathogenesis of this entity, it is not commonly used. We present a case report of oral fibroma in the left side of the buccal mucosa in 50 years old Saudi patient.
\end{abstract}

Keywords: Fibroma, buccal mucosa, tumor.

\section{Introduction}

Fibroma is a benign neoplasm of fibroblastic origin and it is rare in the oral cavity (Scully, 2010). It was discovered that majority of the fibromas occurring in the oral cavity are reactive in nature and represent a reactive hyperplasia of fibrous connective tissue in response to local irritation or trauma rather than being a true neoplasm (Barker et al.,1967). However, Stout stated that "it is exceedingly difficult to decide whether or not there is true benign neoplasm composed of fibroblasts" (Shamim et al., 2008). Since true fibromas of oral and maxillofacial areas are infrequent (Goravalingappa et al., 1999), the case below is one of its kinds. Consequently, the histologic criteria of a true fibroma were first described by Barker and Lucas. Three more cases of true gingival fibroma have been reported in literature since then 
(Christopoulos et al., 1994). Also, we describe a similar case of a true fibroma in the left side of alveolar mucosa.

A 50 years old Saudi male was referred to the Dental Clinic of the College of Dentistry at Al-Jouf University in Sakaka, Saudi Arabia. Thus, the patient reported the presence of a ball in his mouth approximately six months ago. He complained of wounds on the tissue while eating. This painless ball became ulcerated, bleeding and with pain. Nevertheless, the complete medical history revealed no systemic diseases.
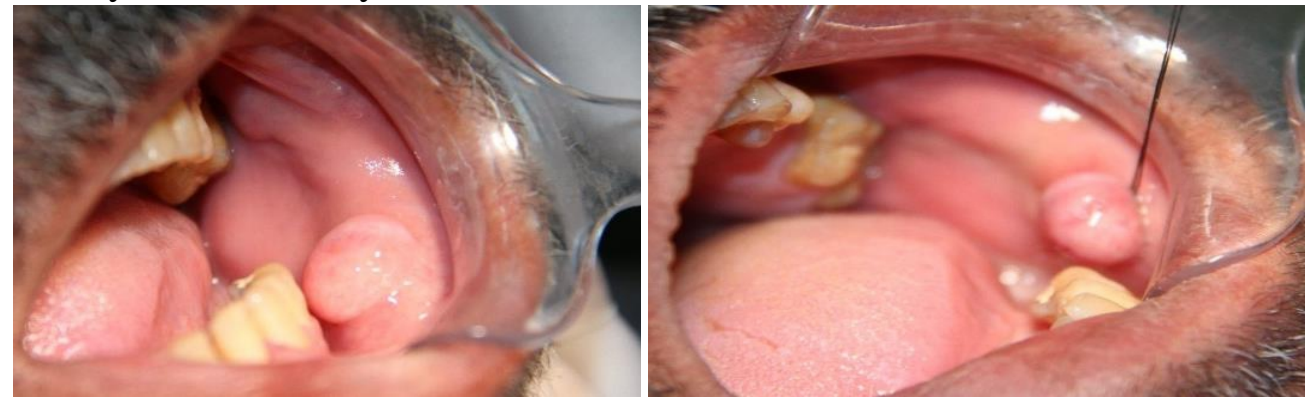

Figure $1 \&$ 2. Intraoral examination revealed a soft nodule in the premolar left side

The intraoral examination revealed a soft nodule in the left side which was covered by normal mucosa. Intra orally on palpation, it was ovoid and firm in terms of consistency and is located in the premolar-molar region (Figure $1 \& 2$ ).

Radiographic examination no calcifications. Irritation fibroma, neurofibroma, peripheral ossifying fibroma, and benign tumors of nerve and muscle origin were provisionally diagnosed. The encapsulated lesion, noted to be located between periosteum and alveolar mucosa, was excised under local anesthesia and was sent for histopathological evaluation.

In the pre-surgical preparation, all the normal range of preoperative routine investigations was collected. They include; complete blood count, coagulation tests, and blood pressure. However, the patient underwent surgical excision of the lesion under local anesthesia on the dental chair.

Local infiltration anesthesia around the lesion mepivacaine $(3 \%)$ with vasoconstrictor was injected. This was followed by incision with a scalpel blade $n^{0} 15$, giving the margin of the lesion, and grasped with mosquito and tissue forceps to complete surgical resection (Figure 3). After the removal of he lesion, then the incision was sutured with 3-0 black silk suture (Figure 4). 

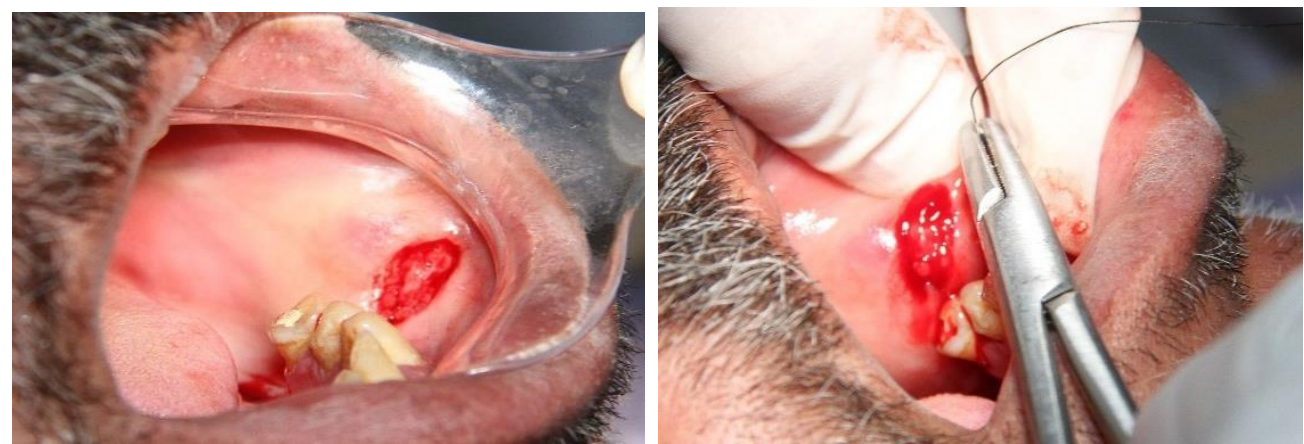

Figure $3 \& 4$. Surgical resection and suturing

The piece was placed in a pot with $10 \%$ formaldehyde where it floated, showing the diagnosis of the specimen. Then it was sent for pathological examination which appears as soft, yellowish, and the size of the lesion was approximately $11 * 10 \mathrm{~mm}$ in diameter (Figure 5). Here, the material was processed and examined, thereby confirming the diagnosis of fibroma of the classical type.

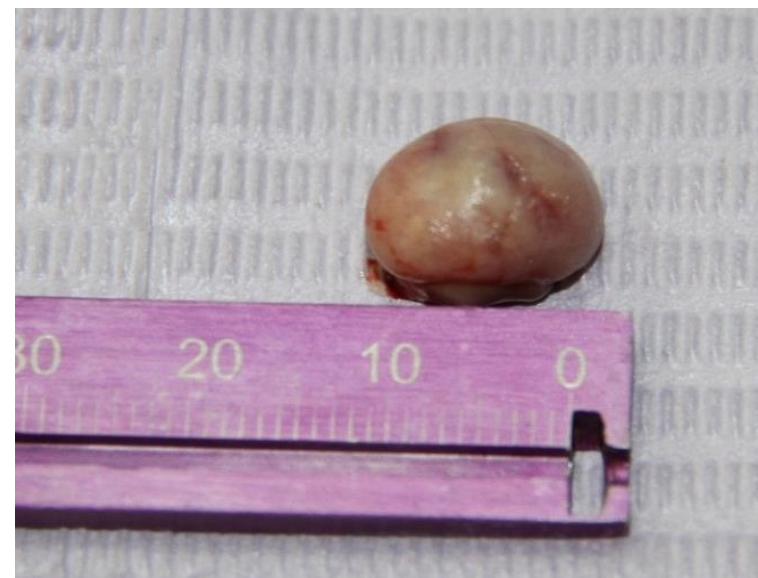

Figure 5. Removal of the lesion $(11 * 10 \mathrm{~mm}$ in diameter $)$

\section{Histopathology}

Histopathology revealed a fattened hyper-orthokeratotic stratified squamous epithelium overlying a well circumscribed encapsulated mass of dense collagenous stroma. The stroma was composed of numerous spindles shaped plump fibroblasts, collagen arranged in parallel, or interlacing dense bundles with areas of hyalinization, sparse inflammatory cells, and minimal vascularity (Figure $6 \& 7$ ).

This low-power photomicrograph illustrates that the fibrosis is an encapsulated lesion generally covered by intact but somewhat thin squamous epithelium. Even at this power, one can see the fairly dense fibrous tissue extending into the submucosal area. In addition, a higher magnification shows 
the extremely dense wavy collagen bundles containing few fibroblasts. Also, there is little or no inflammation and the epithelium covering the convex surface is intact (Figure 8).

At high-power, the growth shows extremely broad collagen bundles with a small blood vessel in the central portion. Spindle-shaped fibroblasts are sparse and do not have inflammations. If this were a true fibroma or neoplasm of fibroblasts, one would expect to see many cells and much less collagen. Although prognosis is excellent, a diagnosis of "True Fibroma" was demonstrated (Figure 9).

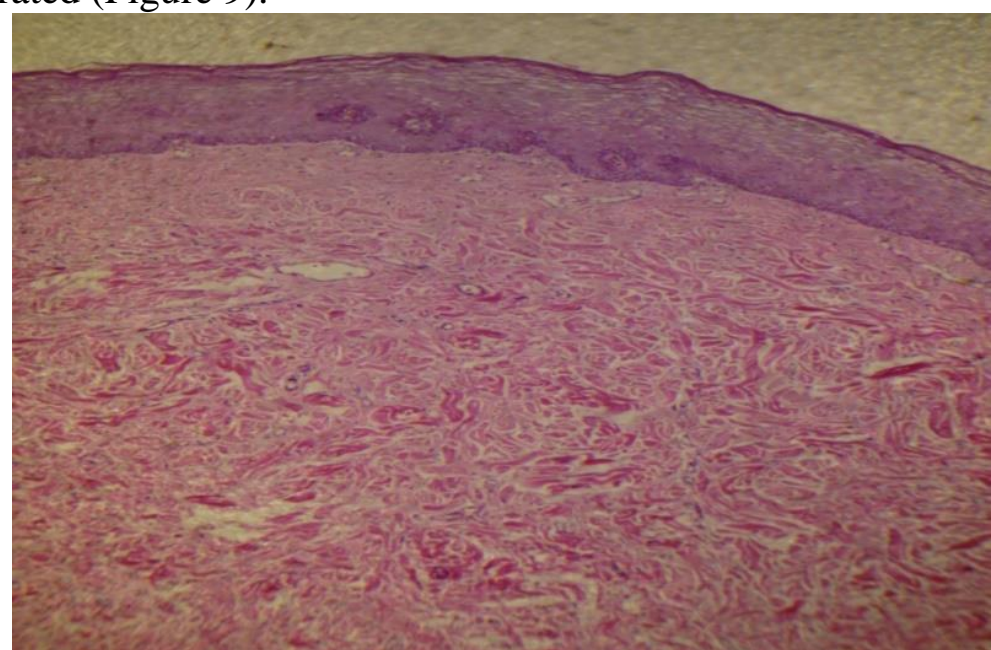

Figure 6. Low-power view showing fibroma. An orthokeratinized stratified squamous atrophic epithelium with loss of flat rete-ridge pattern underlying dense Connective tissue stroma was reported. The CT contains dense bundles of collagen fibers and moderate number of fibroblasts and fibrocytes along with little chronic inflammatory cells $(\mathrm{H} \& \mathrm{E}$ stain X 100)

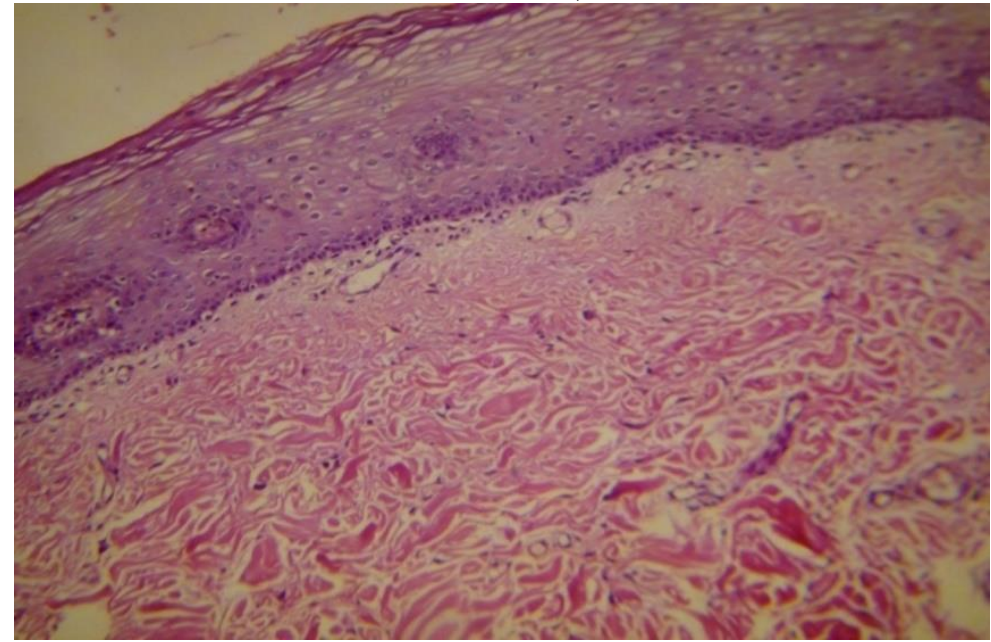

Figure 7. High-power view of previous slide showing fibroma with dense bundles of collagen fibers and moderate number of fibroblasts and fibrocytes along with little chronic inflammatory cells (H \& E stain X 200) 


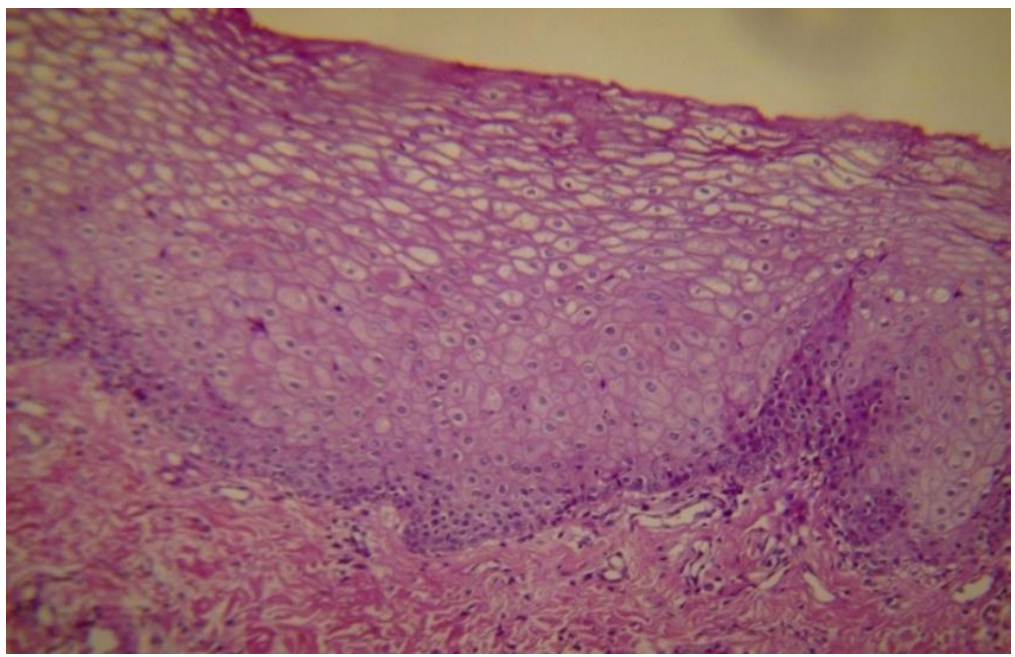

Figure 8. High-power view histopathologic of previous slide showing a orthokeratinized stratified squamous atrophic epithelium with flat rete-ridge pattern covering a fibrous connective tissue stroma was reported (H \& E stain X 400)

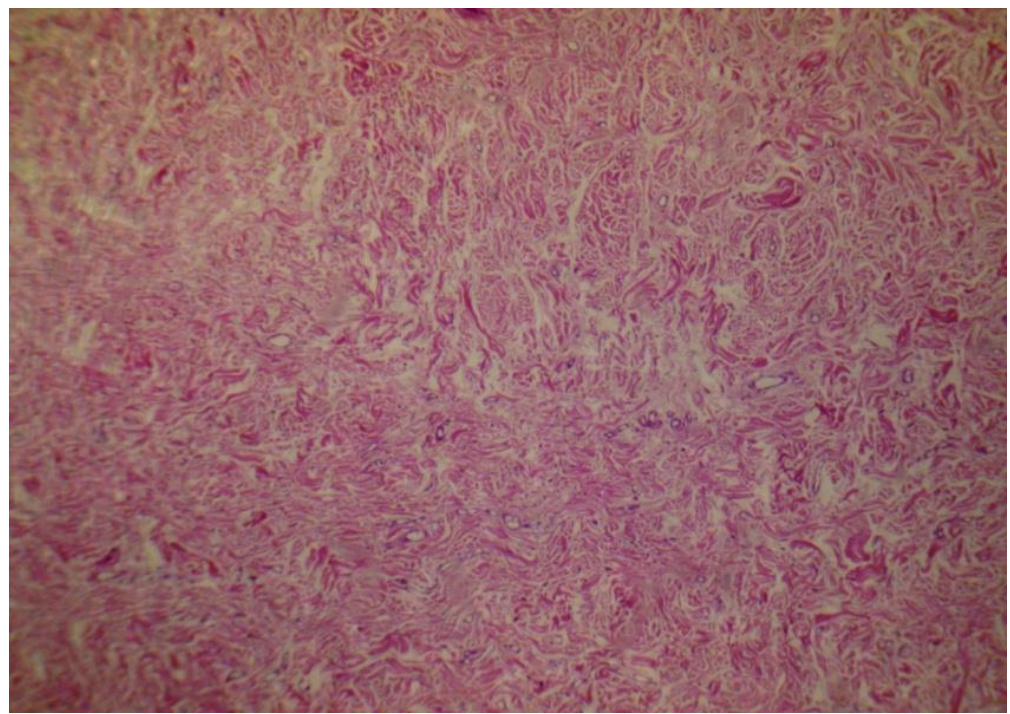

Figure 9. Higher-power view of previous slide showing C.T. which depicted dense bundles of haphazardly placed collagen fibers along with fibroblasts and few chronic inflammatory cells. An increased number of dilated blood vessels in the lamina propria (H \& E stain X 400)

\section{Discussion}

As far as the nature of these lesions goes, most pathologists believe that both hyperplasia's and neoplasms can occur. However, it is very difficult to differentiate between the two and decide whether a benign neoplasm exists or not. The extremely low frequency of the occurrence of true fibromas (benign neoplasms) was highlighted by Barker et al in 1967. In their study, they reported two true fibromas among 171 specimens of localized fibrous 
growths (Barker \& Lucas, 1967; Christopoulos, Sklavounou, \& Patrikiou, 1994). A reactive or irritational fibroma usually has an etiology, that is, a source of irritation. On the other hand, benign fibrous neoplasms do not have that. Also, the character of these lesions tells a story. According to Barker and Lucas, irritational fibromas exhibit a pattern of collagen arrangement depending on the amount of irritation and the site of the lesion.

Furthermore, there are two types of patterns: (a) radiating pattern and (b) circular pattern. In the radiating type, the fires radiate towards the epithelium from the base of the lesion. However, the circular type shows a central mass of disoriented fires surrounded by a peripheral layer of collagen fires running beneath and parallel to the overlying epithelium. Thus, they hypothesized that the former appears when there is a greater degree of trauma and in sites which are immobile in nature (e.g., palate). Consequently, lesser trauma induces the latter and it occurs in sites that are flexible in nature (e.g., cheeks). True fibromas can be differentiated from normal tissue which involves the presence of a capsule. The present case also showed histopathological features suiting the above-mentioned criteria, pointing towards the diagnosis of a true fibroma.

\section{References:}

1. Barker D. S. \& Lucas R. B. (1967). "Localized fibrous overgrowths of the oral mucosa," British Journal of Oral Surgery, vol. 5, no. 2, p. 8692.

2. Christopoulos P., Sklavounou A., \& Patrikiou A. (1994). "True fibroma of the oral mucosa: a case report," International Journal of Oral and Maxillofacial Surgery, vol. 23, no. 2, p. 98-9.

3. Goravalingappa J. P. \& Mariyappa K. C. (1999). "Fibroma of tonsil," Indian Journal of Otolaryngology and Head and Neck Surgery, vol. 51, no. 3, p. 72-3.

4. Scully C. (2010). Oral Medicine and Pathology at a Glance, Wiley Blackwell, 1st edition.

5. Shamim T., Varghese V. I., Shameena P. M., \& Sudha S. (2008). “A retrospective analysis of gingival biopsied lesions in south Indian population," Medicina Oral, Patologia Oral y CirugiaBucal, vol. 13, no. 7 , p. 414-8. 